\title{
Advantages of CCD detectors for de novo three-dimensional structure determination in single-particle electron microscopy
}

\author{
B. Sander, M.M. Golas, H. Stark * \\ Research Group of 3D Electron Cryomicroscopy, Max Planck Institute for Biophysical Chemistry, Am Fassberg 11, 37077 Göttingen, Germany
}

Received 16 March 2005, and in revised form 20 April 2005

Available online 23 May 2005

\begin{abstract}
For three-dimensional (3D) structure determination of large macromolecular complexes, single-particle electron cryomicroscopy is considered the method of choice. Within this field, structure determination de novo, as opposed to refinement of known structures, still presents a major challenge, especially for macromolecules without point-group symmetry. This is primarily because of technical issues: one of these is poor image contrast, and another is the often low particle concentration and sample heterogeneity imposed by the practical limits of biochemical purification. In this work, we tested a state-of-the art $4 \mathrm{k} \times 4 \mathrm{k}$ charge-coupled device (CCD) detector (TVIPS TemCam-F415) to see whether or not it can contribute to improving the image features that are especially important for structure determination de novo. The present study is therefore focused on a comparison of film and CCD detector in the acquisition of images in the low-to-medium ( 10-25 $\AA$ ) resolution range using a $200 \mathrm{kV}$ electron microscope equipped with field emission gun. For comparison, biological specimens and radiation-insensitive carbon layers were imaged under various conditions to test the image phase transmission, spatial signal-to-noise ratio, visual image quality and power-spectral signal decay for the complete imageprocessing chain. At all settings of the camera, the phase transmission and spectral signal-to-noise ratio were significantly better on CCD than on film in the low-to-medium resolution range. Thus, the number of particle images needed for initial structure determination is reduced and the overall quality of the initial computed 3D models is improved. However, at high resolution, film is still significantly better than the CCD camera: without binning of the CCD camera and at a magnification of $70 \mathrm{k} \times$, film is better beyond $21 \AA$ resolution. With 4-fold binning of the CCD camera and at very high magnification $(>300 \mathrm{k} \times)$ film is still superior beyond $7 \AA$ resolution.
\end{abstract}

(c) 2005 Elsevier Inc. All rights reserved.

Keywords: CCD; Electron microscopy; Single-particle analysis; Structure

\section{Introduction}

Projection images of macromolecular complexes obtained by electron cryomicroscopy contain in principle the information needed for the computation of their three-dimensional (3D) structure. Problems in the de novo determination of the $3 \mathrm{D}$ structure often arise from uneven angular distribution and a biochemically unavoidable degree of heterogeneity of the sample. In

\footnotetext{
* Corresponding author. Fax: +4905512011197.

E-mail address: hstark1@gwdg.de (H. Stark).
}

this context, two factors in particular contribute significantly to the difficulty of 3D structure determination. One is loss in image quality (such as poor image contrast), and the other is low particle statistics due to limited concentration of the sample.

In the first steps of de novo single-particle image analysis, the individual particle images have to be aligned properly, to obtain images with improved signal-to-noise ratio (SNR). By the application of multivariate statistical analysis (MSA) followed by classification of images (Frank, 1996; van Heel, 1984; van Heel and Frank, 1981), image classes representing similar views of the molecules become recognisable. The alignment 
algorithms commonly used for this task perform well, even at very low SNR, if reliable reference images (e.g., computed from a 3D structure) are already available (Joyeux and Penczek, 2002). However, for determining a totally unknown 3D structure of a macromolecular complex, reference images computed from an earlier 3D density are not available. In the absence of 3D models, "reference-free" alignment approaches (Dube et al., 1993; Penczek et al., 1992) have to be applied, in order to obtain first particle averages. In the initial image-processing phase, the power of intermediate reference images to drive alignment and classification of the images is obviously much lower than it is in later steps of the image analysis. Therefore, high SNRs are required for successful image-processing. Obviously, the reliability of any 3D structure computed from two-dimensional class averages is strongly dependent on the correctness of the first two-dimensional steps in image-processing. Such class averages normally exhibit structure information up to $\sim 20 \AA$. Thus, to obtain a reliable $3 \mathrm{D}$ structure, the information content of raw images in the resolution range where initial $2 \mathrm{D}$ analysis takes place is of great importance. Tests to compare the performance of CCD detectors with photographic film are required - not only in the range of very high resolution (where film is usually better than CCD), but also at low and intermediate spatial frequencies.

Traditionally, conventional silver halide film has been favoured for single-particle data collection, as the procedure is faster and allows the recording of large numbers of particle images in a short time. CCD detectors, in contrast, have the intrinsic disadvantage of low readout speed, coupled with small pixel numbers that limit the speed and efficiency of data collection. So far, CCD detectors have widely been used to record high-resolution electron diffraction patterns of two-dimensional protein crystals (Bullough and Henderson, 1999; Subramaniam et al., 1999), and their applicability for singleparticle 3D work (Booth et al., 2004; Stewart et al., 2000; Zhang et al., 2003) and for high tensions up to $400 \mathrm{kV}$ (Brink and Chiu, 1994; Downing and Hendrickson, 1999) has been demonstrated. Currently, CCD cameras are equipped with very efficient polycrystalline phosphor scintillators and cooled to $\approx-30^{\circ} \mathrm{C}$, to lower the readout noise (Fan and Ellisman, 2000; Faruqi and Subramaniam, 2000). As a consequence, CCD detectors offer a higher SNR at low resolutions than film does (Booth et al., 2004). Therefore, the use of CCD detectors may nevertheless be worth the extra effort when the image quality of conventional film is inadequate because of low image contrast.

Routinely, the signal decay (Saad et al., 2001) is determined from power spectra of the electron-microscopic images as a first estimate of the quality of signal transmission in terms of a $B$ factor. This means that the amplitude information is used to estimate the quality of signal transmission. However, for computerised imageprocessing, the phase information is of paramount importance, and yet it is not possible to describe the quality of image phases at a certain spatial frequency from the $B$ factor without further experimental evidence (Sherman and Chiu, 1997). In our experience, derived from many de novo 3D structure determinations of large (asymmetrical) macromolecular complexes, image alignment together with MSA-based classification is indeed more reliable when a CCD camera is used instead of film (Golas et al., 2005). Another advantage of the CCD camera is the smaller number of particle images needed to obtain first meaningful results. The aim of this work was thus to find an explanation for this practical observation, and to discover why images recorded on a CCD camera are indeed superior for the initial image-processing in single-particle electron cryomicroscopy. Two different approaches were applied to test the reliability of image phase transmission of a $4 \mathrm{k} \times 4 \mathrm{k} C \mathrm{CD}$ camera and film. First of all, we measured the reliability in image phase transmission by recording series of images from identical areas on a carbon support layer on photographic film or with a CCD camera. After pairwise alignments within each image series, the phase similarity was measured by means of the differential phase residual. The signal from the amorphous sample allowed determination of phase transmission reliability and spectral signal-to-noise ratio (SSNR) in a manner that was independent of radiation damage, but took account of the influence of image alignment. Secondly, images of negatively stained tobacco mosaic virus (TMV) were recorded, and the first steps of single-particle analysis were performed. In this case, the ability of the computer averaging procedure to recover the characteristic helical features in the range of $11.5-23 \AA$ was determined from the power spectra.

\section{Materials and methods}

\subsection{Specimen preparation}

For comparable measurements of signal transmission independent of radiation damage, a single carbon film mounted on a copper grid was imaged directly. For specimen preparation of TMV, a solution of TMV in a buffer containing $200 \mu \mathrm{g} / \mathrm{ml}$ virus, $20 \mathrm{mM}$ Hepes and $150 \mathrm{mM}$ $\mathrm{NaCl}$ was placed in a Teflon well. The specimen was prepared according to a protocol recently published (Golas et al., 2003). Briefly, a carbon film evaporated onto freshly cleaved mica was floated on the surface of the solution allowing adsorption of virus over $2 \mathrm{~min}$. The carbon film was then transferred to a second well filled with $2 \%$ uranyl formate solution and incubated for $2 \mathrm{~min}$. Subsequently, the carbon film with adsorbed particles was attached to a 400 mesh copper grid on which a 
carbon film containing holes of $\sim 1 \mu \mathrm{m}$ diameter had previously been mounted. Finally, another carbon film floated onto a second solution of uranyl formate was used to form a sandwich, thus enclosing the specimen in a layer of staining solution between two carbon films. The TMV specimens were stored under dry conditions until image acquisition.

\subsection{Electron microscopy}

Images were taken on a $200 \mathrm{kV}$ transmission electron microscope (TEM) equipped with a field emission gun (CM200 FEG, Philips, Eindhoven, The Netherlands) and constantly operated at $200 \mathrm{kV}$ keeping the sample at room temperature. A $4 \mathrm{k} \times 4 \mathrm{k} 16$ bit CCD camera (TemCam-F415, TVIPS, Gauting, Germany) with $15 \mu \mathrm{m}$ pixel size and polycrystalline phosphor scintillator (high sensitivity, optimised for $200 \mathrm{kV}$ ) cooled to $-25^{\circ} \mathrm{C}$ was used. The post-magnification factor of the CCD camera was found to be 1.37- to 1.4-fold with respect to previous calibration using TMV specimens. Camera settings included $1 \times, 2 \times$, and $4 \times$ binning of the pixels to yield effective pixel side lengths of 15,30 , and $60 \mu \mathrm{m}$. The mean number of counts per pixel was adjusted to an electron dose of about $20 \mathrm{e}^{-} / \AA^{2}$ for a single exposure, and ranged from about 2800 to 19000 counts dependant on magnification and CCD pixel binning. For a complete list of settings applied, see Table 1. For comparison with film, images were recorded on Kodak SO-163 film at a magnification of $50000 \times$, developed in Kodak D19 at $20^{\circ} \mathrm{C}$ for $12 \mathrm{~min}$ and digitised on a drum scanner with photomultiplier (Tango, Heidelberger Druckmaschinen, Heidelberg, Germany) at the maximum optical resolution of $6350 \mathrm{dpi}$, and then coarsened by 2 -fold binning, leading to a sampling interval of $1.6 \AA /$ pixel. For the CCD camera, TMV images were recorded with slight overlap of $\sim 15 \%$ so that a continuous rectangular area of $\sim 1 \mu \mathrm{m}^{2}$ could be obtained by precise cross-correlation-based stitching of the single images (TVIPS EM-MENU software, Gauting, Germany). All images of carbon film and TMV were taken at defoci of about $-1 \mu \mathrm{m}$. Additional test images of carbon/gold have been recorded for comparison and are available on request.

\subsection{Measuring the reliability of phase transmission by using images of carbon support film}

Fifty images of the same area of carbon film were recorded on film and on CCD for each setting of the CCD camera. Owing to minor drift of the sample holder, the images within each series had to be aligned by using the "direct alignment" algorithm from Imagic-5 (van Heel et al., 1996). For all alignment operations, the images were slightly high-pass filtered and normalised. The images were used to compute the differential phase residual (DPR), spatial signal-to-noise ratio (SSNR) and power-spectral signal decay as follows: to measure the DPR between two consecutively recorded images, images of $800 \times 800$ pixels were used. Image no. $n+1$ of the series was aligned against image no. $n$ for $n=1 \ldots 49$ to yield 49 aligned pairs of carbon film images. By using the Imagic "Fouring" routine, the DPR between the two single images of each pair was computed. Finally, all results for $n=\{1 \ldots 49\}$ were averaged leading to an almost negligible standard deviation of the mean $\left(<1^{\circ}\right)$. Pixel frames smaller than $800 \times 800$ pixels (e.g., $256 \times 256$ ) were also used to verify that the results were independent of the size of the carbon film area; only the 'smoothness' of the DPR curves was reduced at high spatial frequencies, when smaller frames were applied. For further analysis and interpretation, the minima of DPR (at the positions of the $\mathrm{CTF}^{2}$ maxima) were connected by interpolation (see below).

Table 1

List of all tested settings of the CCD camera, stating effective magnification, pixel binning of the CCD camera, mean number of counts used to achieve an electron dosage of $\sim 20 \mathrm{e}^{-} / \AA^{2}$, and maximum imageable area on the specimen level

\begin{tabular}{|c|c|c|c|c|c|c|}
\hline $\begin{array}{l}\text { Magnification } \\
\left(10^{3} \text {-fold }\right)\end{array}$ & $\begin{array}{l}\text { Binning } \\
(n \text {-fold })\end{array}$ & Mean counts & Nyquist freq. $^{-1}(\AA)$ & $\begin{array}{l}\text { Max. side } \\
\text { length }(\mathrm{nm})\end{array}$ & $\begin{array}{l}\text { No. of images } \\
\text { to cover area of film }\end{array}$ & $\begin{array}{l}\text { DPR } \\
\text { quality limit }(\AA)\end{array}$ \\
\hline 343 & 4 & 4250 & 3.5 & 179 & 130 & 6 \\
\hline 279 & 4 & 6450 & 4.3 & 220 & 88 & 7 \\
\hline 214 & 4 & 10950 & 5.6 & 287 & 48 & 8 \\
\hline 161 & 4 & 19350 & 7.5 & 384 & 30 & 11 \\
\hline 214 & 2 & 2750 & 2.8 & 287 & 48 & 8 \\
\hline 161 & 2 & 4850 & 3.7 & 379 & 30 & 9 \\
\hline 122 & 2 & 8450 & 4.9 & 502 & 20 & 13 \\
\hline 91 & 2 & 15150 & 6.6 & 676 & 12 & 18 \\
\hline 91 & 1 & 3800 & 3.3 & 676 & 12 & 17 \\
\hline 70 & 1 & 6400 & 4.3 & 881 & 6 & 21 \\
\hline
\end{tabular}

Last column: resolution at which the Kodak SO-163 film transmitted image phases are better than the CCD camera when the predicted minimal DPR curves of film and CCD camera were compared.

a Assuming a magnification of $50000 \times$ and a film area of $9 \times 12 \mathrm{~cm}$ or $1.8 \mu \mathrm{m} \times 2.4 \mu \mathrm{m}$ at the specimen size. If the CCD images are recorded with a slight overlap to allow for later stitching, the number will be higher dependent on the proportion of redundantly recorded areas. 
For calculation of SSNR, a procedure identical to that of TMV image-processing (see below) was used: eight consecutively recorded images of carbon film representing an identical area of about $470 \times 470 \AA$ were aligned against one of the eight images that served as reference. Thereafter the eight aligned images were averaged. The SSNR of the eight images with respect to the average was computed as described previously (Unser et al., 1987). To verify the statistical significance of the results, this procedure was also performed for six independent sets of eight images.

The power-spectral signal decay of the images was determined from the rotationally averaged power spectrum of one representative image of size $1024 \times 1024$ pixels. A fitting procedure similar to that described earlier (Sander et al., 2003a) was applied to the rotationally averaged power spectrum in order to determine simultaneously the defocus, an exponential signal decay function connecting the maxima, and a noise function connecting the minima. In addition, these parameters were estimated by applying a quasi-Newton-based fitting routine (R-Software, 2004). No relevant differences between the two methods were found. Finally, the power-spectral signal decay in the important resolution range from $1 / 20$ to $1 / 10 \AA^{-1}$ was calculated from the fit of the envelope functions.

\subsection{Image-processing of $T M V$}

For TMV image-processing, TMV fibres were selected manually from the images so that about $200-300$ sub-windows of $470 \times 470 \AA$ were obtained. Images of TMV were corrected for defocus and astigmatism as described previously (Sander et al., 2003a), thereby verifying a defocus of about $-1 \mu \mathrm{m}$. All TMV images were normalised to a mean of zero and a standard deviation of 10 , and were band-pass filtered. For band-pass filtering, a constant lower cut-off of $1 / 180 \AA^{-1}$ was applied, while the upper cut-off was adjusted to the Nyquist frequency of the image. Two or three rounds of alignment via polar coordinates (Sander et al., 2003b) and hierarchical ascendant classification (HAC) based on multivariate statistical analysis (MSA) were performed (on average 10 members per class average). For the first alignment, a randomly selected single image was used as reference, and then the class average, where the typical repetitive motifs of TMV were most clearly visible, was selected as reference. After the result was stable, (1) the average of eight images belonging to the same MSA class and (2) the average of 20 aligned images possessing highest cross-correlation coefficient with the last reference image were computed. In both cases, the power spectrum was analysed for helical features in the range 11.5-23 $\AA$. To investigate the effect of computational pixel binning (coarsening), some images taken at high sampling rates were Fourier-transformed and only the central part up to the desired Nyquist frequency was reverse-transformed. The coarsened images thus obtained were subjected to the alignment and MSA procedures as described above.

\section{Results}

\subsection{Phase transmission reliability using images of carbon support film}

The complete analysis for film and for one of the camera settings tested $(279000 \times$ magnification, $4 \times$ binning) at a constant electron dosage of about $20 \mathrm{e}^{-} / \AA^{2}$ is shown in Fig. 1. As expected, the average differential phase residual function between two subsequently recorded images is minimal (i.e., best phase transmission) at those spatial frequencies where the $\mathrm{CTF}^{2}$ function reaches a maximum. The minima of the DPR, at least in the spatial frequency region of $0.05-0.15 \AA^{-1}$, can be connected by an approximately straight line (Fig. 1A). As plotted in Fig. 2A, the slope and position of that line are practically independent of the defocus in a range from -0.3 up to $-3 \mu \mathrm{m}$, but are highly characteristic of the camera settings used. Therefore, the maximum achievable consistency of phases can be predicted by interpolating the measured minima of the DPR curve, referred to herein as the 'predicted minimal DPR'. At $279000 \times$ magnification and 4-fold binning, the predicted minimal DPR of the CCD was better than that of film at resolutions lower than $7.3 \AA$. In case of the SSNR functions (Fig. 1B), the maxima of SSNR were connected by linear interpolation similar to the analysis of the DPR minima, and the predicted maximum SSNR was extracted from the interpolation curves as a function of spatial frequency. Again, the exponential decay of the predicted maximum SSNR shows only a minor dependence on the level of defocus chosen (Fig. 2B). The predicted maximum SSNR is slightly better at $\sim 15 \AA$, if the images are taken closer to focus. The maximum SSNR (of eight averaged images, see above) at $\sim 25 \AA$ of the CCD camera was about twice as high as that of film, whereas photographic film revealed a better SSNR at high spatial frequencies beyond $1 / 10 \AA^{-1}$ (Fig. 1B). As shown in Fig. 1C, the CCD camera and film have very similar spatial signal decay. In contrast, the function of the unconvoluted background noise has - at lower spatial frequencies - considerably higher values relative to the signal for film than for the CCD camera.

A comparison of all the different camera settings used with film is made in Fig. 3A. In summary, the predicted minimal DPR of the CCD camera at $20 \AA$ was better than that of film under all conditions tested (by a difference of $7.5^{\circ}-10.6^{\circ}$ for $4 \times$ binning of the camera, a difference of $5.2^{\circ}-8.2^{\circ}$ for $2 \times$ binning and a difference of $2.0^{\circ}-6.9^{\circ}$ for no binning). At $15 \AA$, the predicted min- 
A

Kodak SO-163 film, 50kx, 200kV

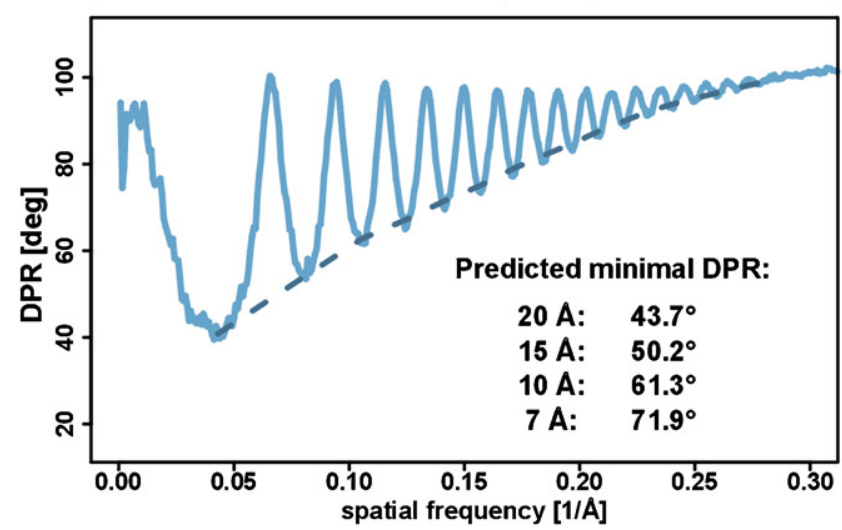

CCD 279kx, 4x binned, 200kV
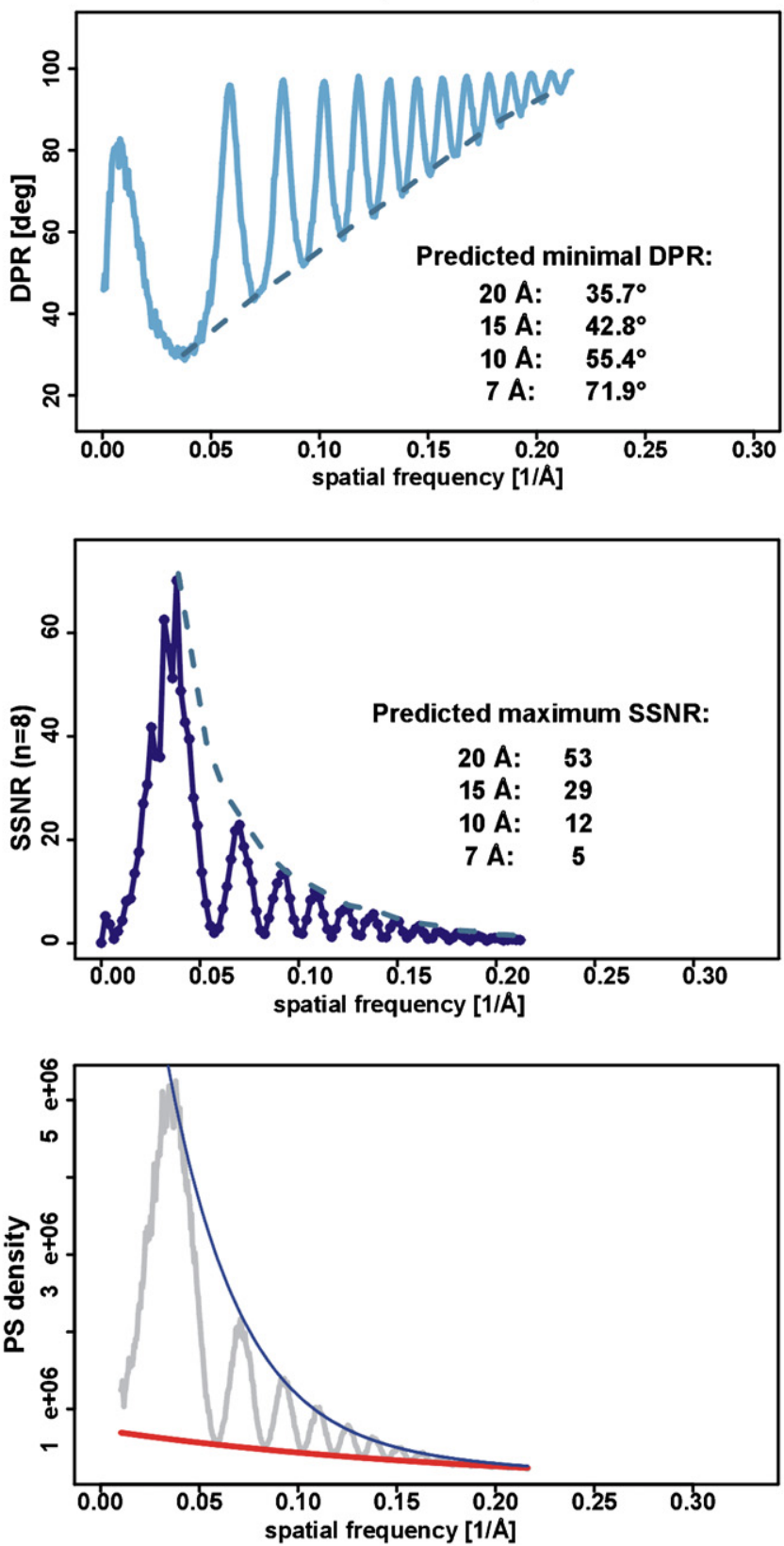

Fig. 1. Analysis of differential phase residual, spatial signal-to-noise ratio and signal decay: comparison between film (left) and one of the CCD camera settings tested (right). (A) Average differential phase residual (DPR) between two consecutively recorded images of amorphous carbon film at a defocus of $-1 \mu \mathrm{m}$ and a voltage of $200 \mathrm{kV}$ for Kodak SO-163 film at 50000× magnification (left panel) and the CCD detector at $279000 \times$ magnification with $4 \times$ binned pixels (right panel). The DPR follows the CTF and is lowest at the maxima of the CTF ${ }^{2}$. Therefore, minima of the DPR are connected by a dashed line to allow the interpolation of the defocus-independent minimal predicted DPR values, which are characteristic for the settings used (compare also Fig. 2A). (B) Spatial signal-to-noise ratio (SSNR) of eight aligned and averaged images of carbon film. Again, as the function follows the $\mathrm{CTF}^{2}$, the maxima have been connected to allow linear interpolation of the defocus-independent maximum values of SSNR obtainable at the settings used (compare also Fig. 2B), CCD detector settings as in (A). (C) Power-spectral signal decay (arbitrary density values) of a representative single image obtained by using film (left panel) and CCD detector (right panel), using the same settings as in (A). The rotationally averaged signal is shown in grey. An exponential function connecting the maxima is shown in blue to indicate the defocus-independent signal decay, and a noise function connecting the minima of the power-spectral signal is shown in red. (For interpretation of the references in colour in this figure legend the reader is referred to the web version of this article.)

imal DPR was lower for the CCD detector compared with film for all 4-fold binned settings of the CCD camera (difference: $4.8^{\circ}-8.5^{\circ}$ ) and the 2 -fold binned ones at magnifications above $161000 \times\left(\right.$ difference: $5.7^{\circ}-6.3^{\circ}$ ). In a comparison at the $10 \AA$ level, the CCD camera performed better than film when $4 \times$ binning was used in 

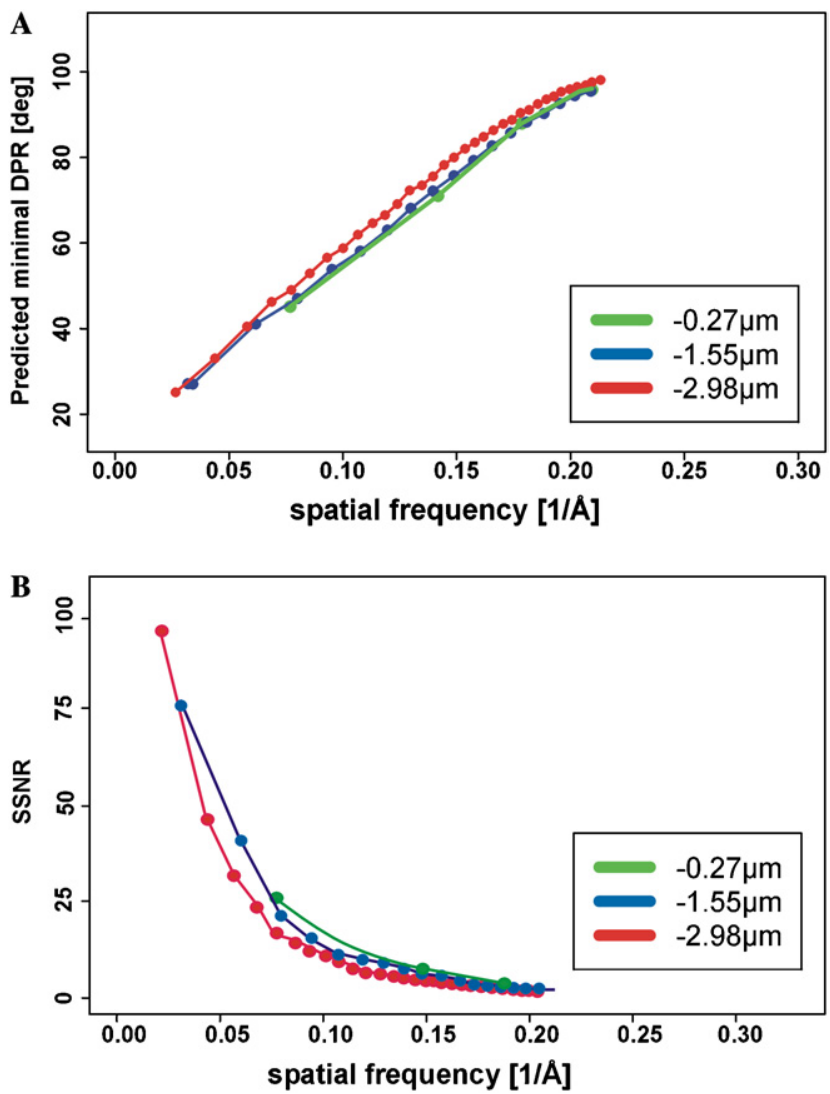

Fig. 2. Dependence of DPR and SSNR on the defocus. (A) Interpolation curves connecting the minima of DPR at three different defoci of $-0.3 \mu \mathrm{m}$ (green), $-1.6 \mu \mathrm{m}$ (blue), and $-3 \mu \mathrm{m}$ (red). The location of the DPR minima is shown by correspondingly coloured dots. The three curves are very close together, indicating that slope and position of the curves are mainly independent of defocus, but are characteristic of the camera settings used. (B) Linearly interpolated maxima of the SSNR for the three different defoci as described in (A). The dots indicate the position of the SSNR maxima. Again, the maximum predicted SSNR shows only a minor dependence on the defocus (slightly better values at around $15 \AA$ for the lower defoci).

combination with magnifications of $\geqslant 214000 \times$ (difference: $3.5^{\circ}-7^{\circ}$ ) or $2 \times$ binning and a magnification of $214000 \times$ (difference: $2.9^{\circ}$ ). At resolutions around $7 \AA$, only the combination of 4-fold binning together with the highest tested magnification of $343000 \times$ led to a better transmission of phases in comparison with film. The spatial frequencies where the predicted minimal DPR of film crosses the curves obtained for the CCD camera are listed in Table 1. For most of the settings, the CCD camera exhibited a significantly better performance at $20 \AA$ and $15 \AA$, while - even with the highest possible magnification of the electron microscope together with 4-fold binning-photographic film retained the phase information better than the CCD camera at resolutions beyond $6 \AA$.

The situation is very similar for the SSNR. The SSNR measured at the first contrast maximum of the CTF at $\approx 25 \AA$ was higher than that of film for all camera set- tings tested (Fig. 3B); for $4 \times$ binning and $2 \times$ binning combined with magnifications higher than $161000 \times$ the maximum SSNR at $25 \AA$ was $80-120 \%$ higher. At 20 and $15 \AA$, the combination of at least 2-fold binning together with magnifications $\geqslant 161000 \times$ led to a significantly improved signal compared with film. In contrast, the CCD camera exhibited lower SNRs than film at resolutions better than $15 \AA$ under most of the conditions tested. Only at the highest tested magnification of $343000 \times$ and 4 -fold binning of the CCD camera were the values for the SSNR comparable to those of film up to $7 \AA$ (Fig. 3B).

In Fig. 4, the decay in the power-spectrum signal for the CCD camera is compared with the corresponding result for film. We have focussed on the resolution range important for standard single-particle analysis by computing a signal decay factor when going from 20 to $10 \AA$. The best value (i.e., the one with the lowest decay factor) can be obtained with film where signal in the power spectrum going from 20 to $10 \AA$ drops by a factor of only 3.6. In contrast, this factor is higher in case of the CCD camera, and increases with decrease in binning and magnification of the electron microscope $(3.8-6.7 \times$ for $4 \times$ binning of the camera, $4.5-10.9 \times$ for $2 \times$ binning of the camera, and $8.9-14.4 \times$ for $1 \times$ binning of the camera; see Fig. 4).

\subsection{Analysis of TMV images}

For all the ten settings of the CCD camera and also for film, TMV was imaged and analysed by standard averaging procedures (see above). When a power spectrum was calculated from the average, a characteristic $\mathrm{X}$-like arrangement of spots in the resolution range of $18 \AA$ (outermost spots) to $23 \AA$ (innermost spots) could be seen. In every figure, the power spectra computed from normalised images are shown with identical interactive grey values of $0-500$ density units to make the level of background noise and the signal comparable. In Fig. 5, the results for Kodak SO-163 film are shown; the results for CCD camera follow in Figs. 6-8 (for $1 \times, 2 \times$, and $4 \times$ binning, respectively).

In general, the visibility of single TM virus is better when the magnification (and thus the Nyquist frequency) is low. The same holds true for the class averages with $n=8$ members, although in every class average the $23 \AA$ spacings of TMV become clearly visible. The power-spectral signal pattern is visible best in the power spectra of the class averages $(n=8)$ for lower magnifications and $\geqslant 2 \times$ pixel binning of the camera. In the power spectra of the class averages $(n=8)$, the background noise increases with finer sampling of the images (e.g., compare the top row with the bottom row in Fig. 7). Whereas these power spectra of the class averages $(n=8)$ mainly differ in the amount of noise present in the $15-25 \AA$ range, the direct averages of 20 

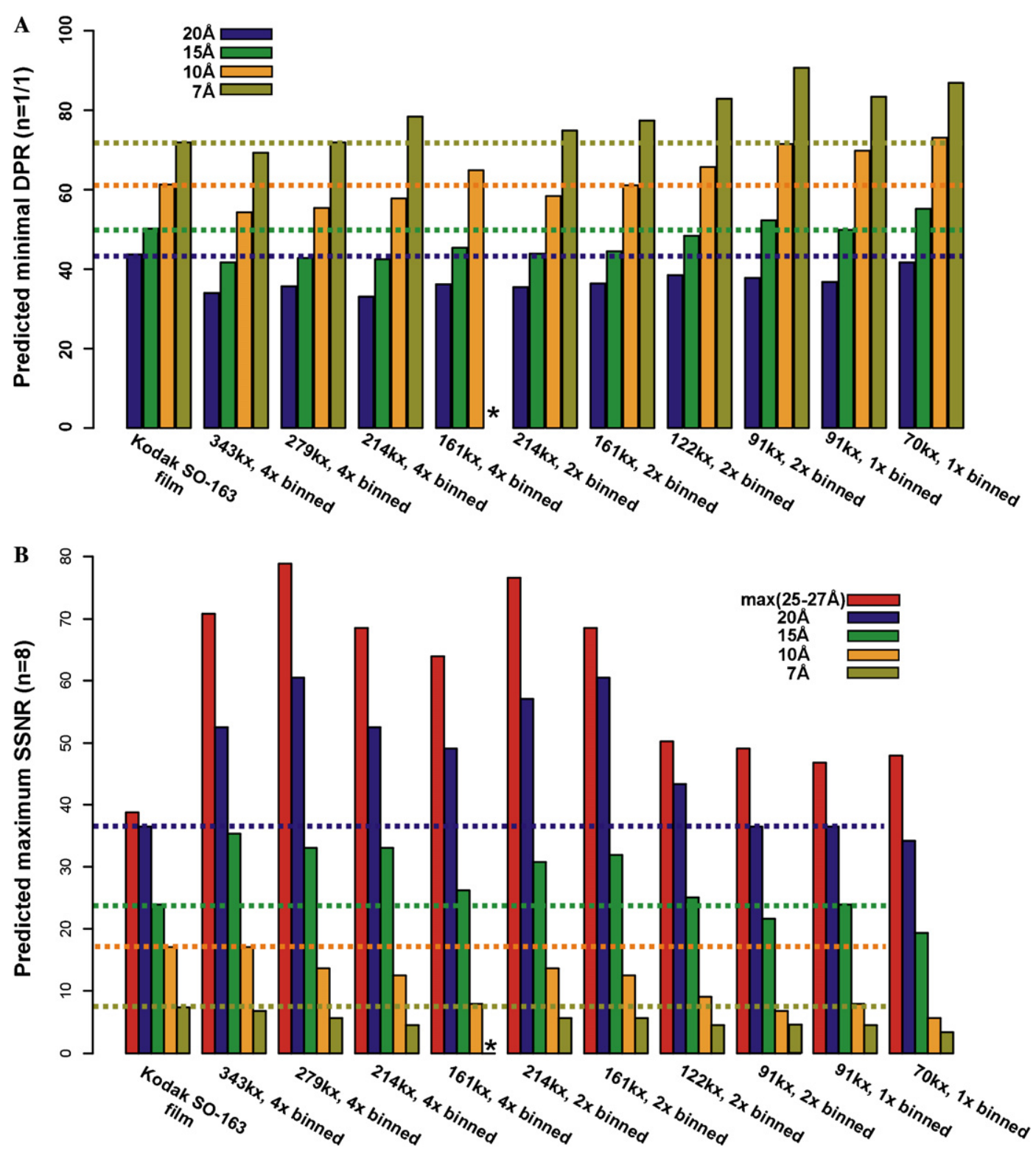

Fig. 3. Summary of DPR and SSNR for all the settings tested. (A) Histogram of all values measured for the predicted minimum DPR (see Fig. 1) for $20 \AA$ (blue), $15 \AA$ (green), $10 \AA$ (orange), and $7 \AA$ (dark yellow). The correspondingly coloured dotted lines indicate the values obtained for Kodak SO-163 film. For a magnification of $161000 \times$ and $4 \times$ binning of the CCD detector, a $7 \AA$ value cannot be obtained (asterisk). (B) Histogram of all values measured for the SSNR of eight aligned and averaged images of carbon film. Red, SSNR at the first maximum (25-27 A); blue, SSNR at 20 ^; green, SSNR at $15 \AA$; orange, SSNR at $10 \AA$; and dark yellow, SSNR at $7 \AA$. Again, the dotted lines show the values obtained for Kodak SO-163 film.

aligned images are virtually noise-free, although they differ in the visibility and intensity of the outer $(\sim 18 \AA)$ spots. In the power spectra of the alignment averages $(n=20)$, the complete $(18-23 \AA)$ helical motif can be seen not only on film (as expected), but also for $2 \times$ binning of the CCD pixels and magnifications $\geqslant 122000 \times$, or $4 \times$ binning of the camera and magnifications $\geqslant 214000 \times$, whereas spots in the $23 \AA$ range are visible for all the settings tested.

When the power spectra of the 20 directly averaged images were displayed with a narrower range of grey values ranging from 0 to 150 (not shown), weak spots at
$11.5 \AA$ became visible for no binning and a magnification of $91000 \times, 2 \times$ binning and magnifications of 91000 $161000 \times$ and $4 \times$ binning and magnifications of $\geqslant 279000$ as well as for film. Only at $4 \times$ binning and a magnification of $343000 \times$ were relatively strong spots at $11.5 \AA$ visible.

\subsection{Interference of sampling interval and cross-correlation-based alignment}

The apparently increasing low-resolution noise in the range of about $20 \AA$, obtained in those cases when finer sampling was applied, does not agree with the fact 


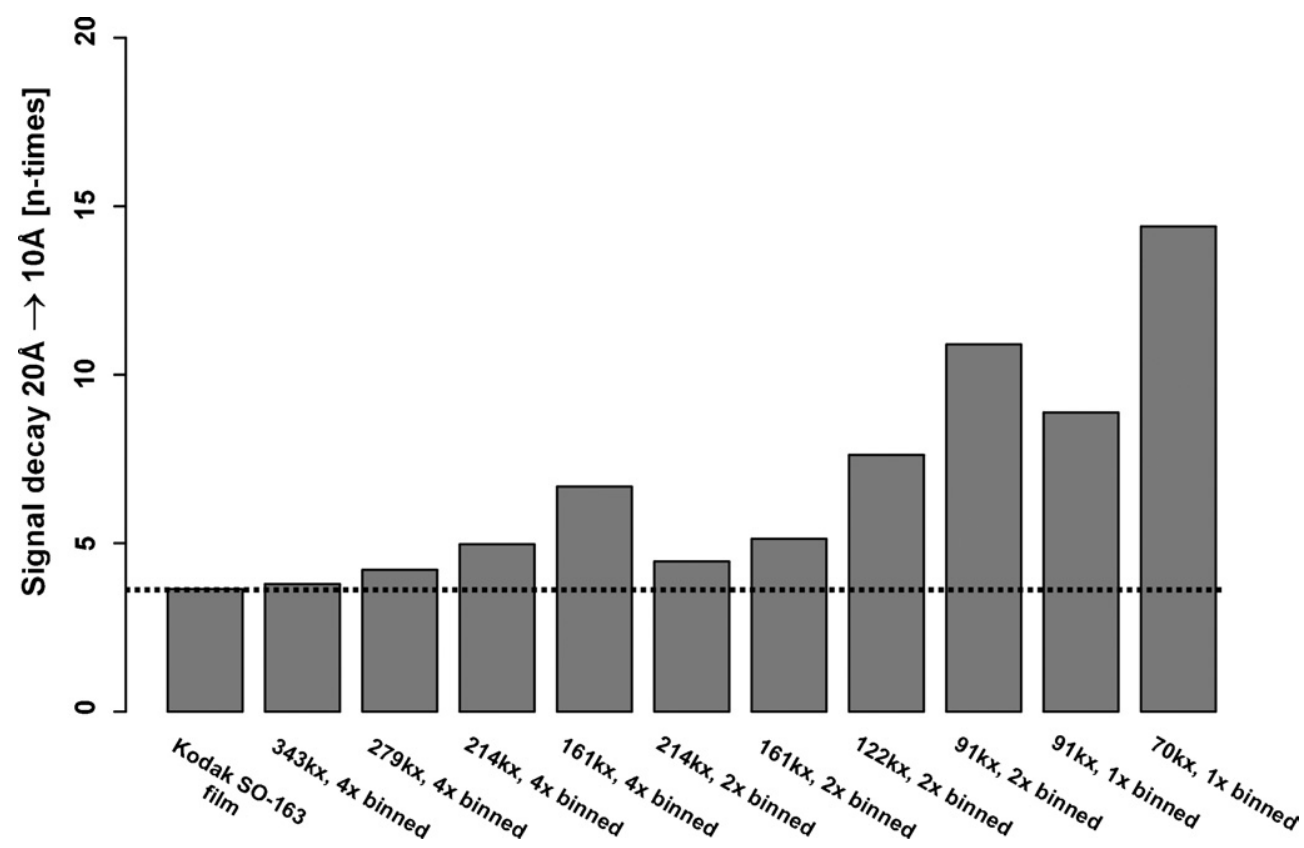

Fig. 4. Power-spectral signal decay obtained from the rotationally averaged power spectrum of a representative image. To allow easy comparison of the resolution range of 10-20 ̊, the signal decay from 20 to $10 \AA$ was calculated and plotted as a histogram. The lowest signal decay can be obtained with film $(3.6 \times$, left bar and dotted line), while with the CCD camera a comparable factor of $3.8 \times$ can only be found for a magnification of $343000 \times$, and $4 \times$ binning; at all other settings the spatial signal decay is considerably higher compared to film.

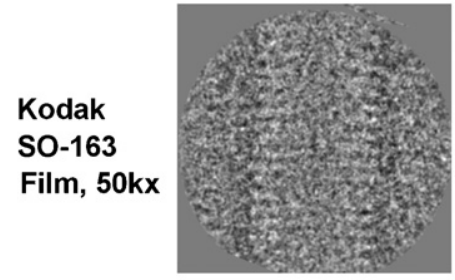

Single image

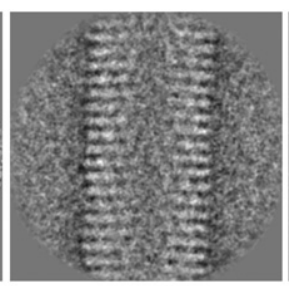

class average $(n=8)$

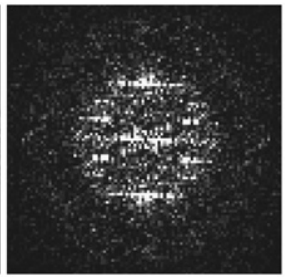

PS ( $n=8)$

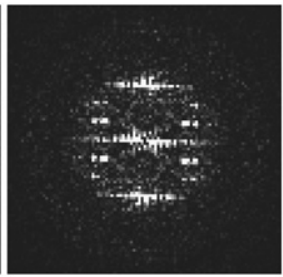

PS $(n=20)$

Fig. 5. Analysis of TMV images recorded on Kodak SO-163 film at $200 \mathrm{kV}, 50000 \times$ magnification and a sampling interval of $1.6 \AA ̊$ pixel. From left to right: single aligned image, MSA-based class average with eight members, power spectrum of that class average, power spectrum of an average image from 20 aligned images.

that the SSNR increases with increasing magnification. However, the cross-correlation-based alignment clearly fails to make full use of the high SNR in the low-resolution range, when the images are highly oversampled (i.e., Nyquist frequencies around $1 / 3 \AA^{-1}$ ). The reason may be found in the application of the standard crosscorrelation coefficient: this implies that all spatial frequencies over the whole range of resolution contribute equally to the alignment, leading to competition between the low-frequency signal and the high-frequency noise. This behaviour of the alignment is demonstrated in Fig. 9: in Fig. 9A the CCD camera is used with $2 \times$ binning of the pixels and a very fine sampling (Nyquist frequency $1 / 3.1 \AA^{-1}$ ), while in Fig. 9B the Nyquist frequency is only $1 / 7 \AA^{-1}$, owing to a lower magnification of the electron microscope. Although the SNR around $20 \AA$ should be higher at the settings used to produce Fig. 9A (compare results obtained with car- bon film, right column), the power-spectral spots canin contrast to the results obtained with a lower magnification (Fig. 9B) - hardly be distinguished from noise. When the images from Fig. 9A are computationally coarsened to a Nyquist frequency of $1 / 7 \AA^{-1}$, the alignment and averaging procedure work well and a clear, noise-free power-spectral signal can be obtained (Fig. 7C). The image quality is even better than in Fig. 9B, as predicted by the DPR and SSNR curves obtained with carbon film.

\section{Discussion}

Currently, there is growing evidence that a protein's membership of larger macromolecular complexes is the rule, rather than the exception (Aloy et al., 2004; Forler et al., 2003; Gavin et al., 2002; Jurica and Moore, 2003). 


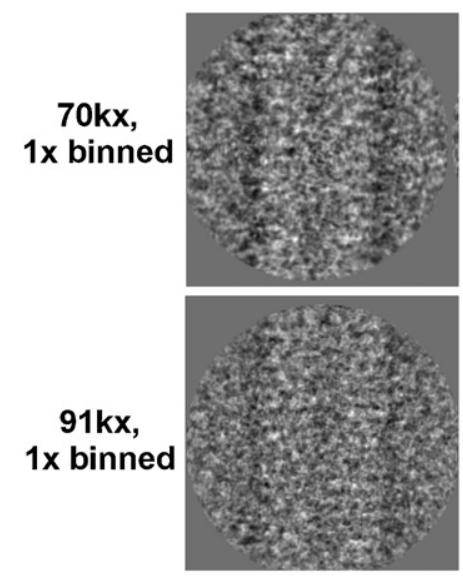

Single image
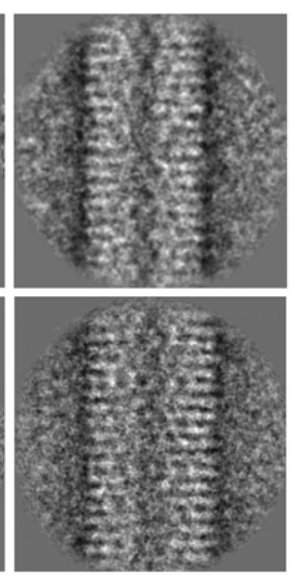

class average $(n=8)$
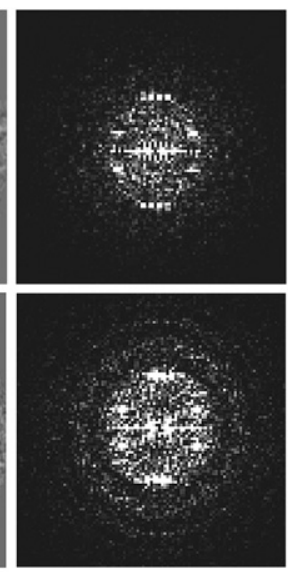

PS ( $n=8)$
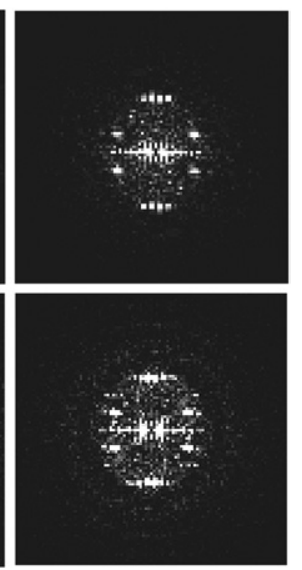

PS $(n=20)$

Fig. 6. TMV images recorded on CCD detector (no pixel binning; pixel size: $15 \mu \mathrm{m}$ ). The mean number of counts has been adjusted to the same electron dose as applied for the film exposure of $\sim 20 \mathrm{e}^{-} / \AA^{2}$ (compare Fig. 5). First row: $70000 \times$ magnification, $2.3 \AA$ 沼el at the specimen level; second row: $91000 \times$ magnification, $1.75 \AA$ /pixel at the specimen level. Columns from left to right: single aligned image, MSA-based class average with eight members, power spectrum of that class average, and power spectrum of an average image from 20 aligned images.

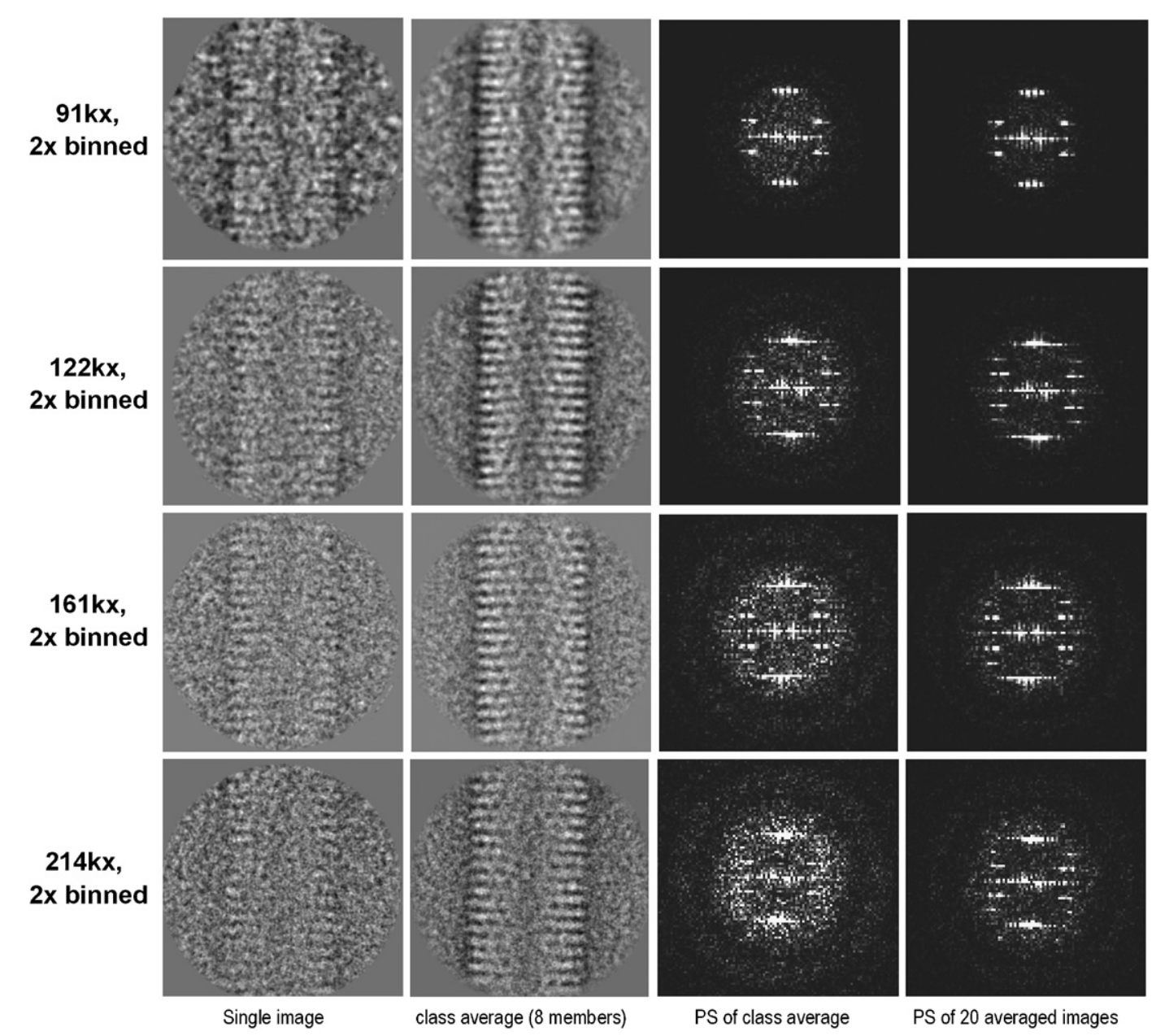

Fig. 7. TMV images recorded on CCD detector ( $2 \times$ pixel binning; pixel size: $30 \mu \mathrm{m})$. The mean number of counts has been adjusted to the same electron dose as applied for the film exposure, i.e., about $20 \mathrm{e}^{-} / \AA^{2}$ (compare Fig. 5). First row, $91000 \times$ magnification, $3.5 \AA$ A pixel at the specimen level; second row, $122000 \times$ magnification, $2.6 \AA /$ pixel at the specimen level; and third row, $161000 \times, 2.0 \AA /$ pixel; last row, $214000 \times, 1.5 \AA /$ pixel. Columns from left to right: single aligned image, MSA-based class average with eight members, power spectrum of that class average, and power spectrum of an average image from 20 aligned images. 


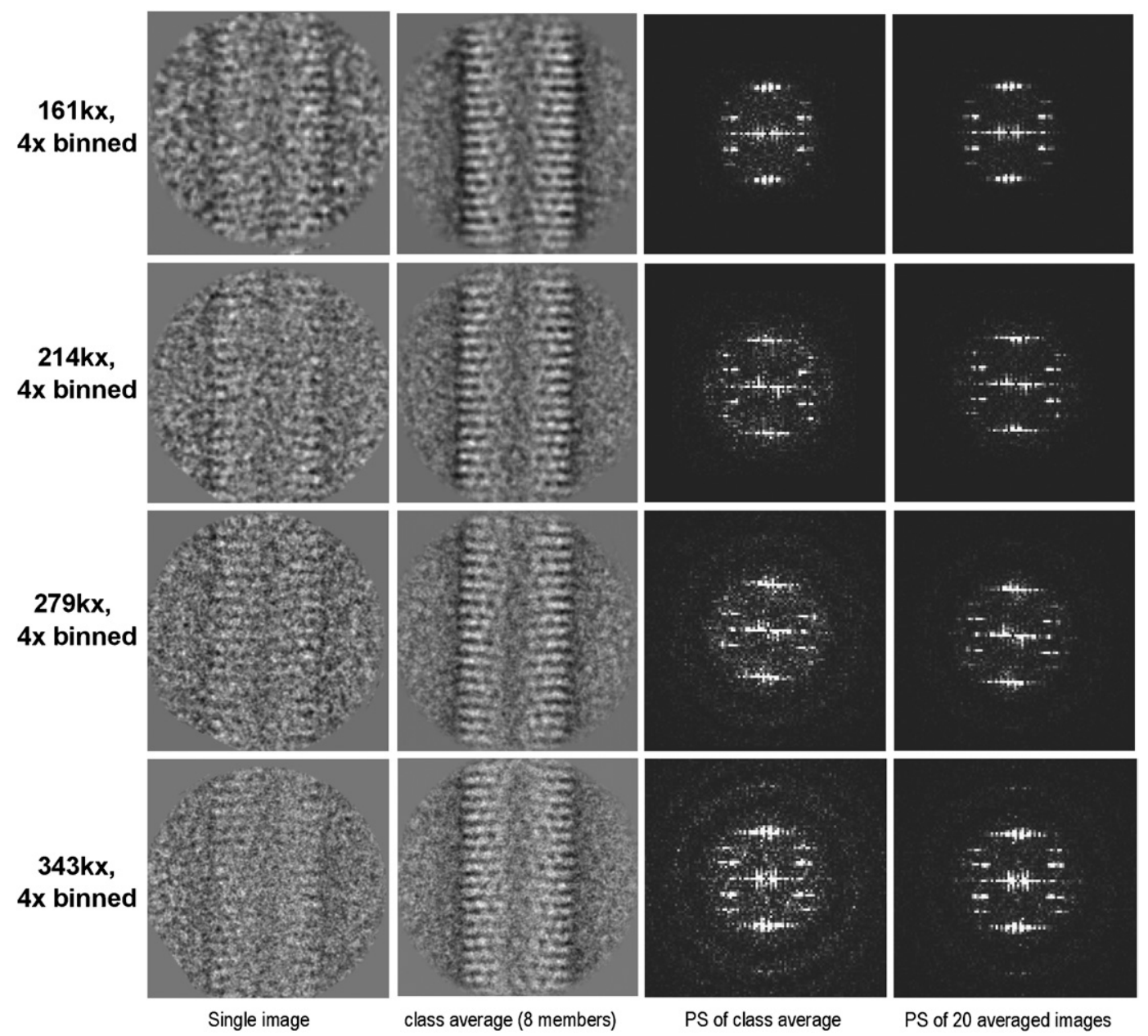

Fig. 8. TMV images recorded on CCD detector ( $4 \times$ pixel binning; pixel size: $60 \mu \mathrm{m})$. The mean number of counts has been adjusted to the same electron dose as applied for the film exposure, i.e., about $20 \mathrm{e}^{-} / \AA^{2}$ (compare Fig. 5). First row, $161000 \times$ magnification, $4 \AA$ /pixel at the specimen level; second row, $214000 \times$ magnification, $3.1 \AA$ Apixel at the specimen level; and third row, $279000 \times, 2.3 \AA$ /pixel; last row, $343000 \times, 1.8 \AA /$ pixel. Columns from left to right: single aligned image, MSA-based class average with eight members, power spectrum of that class average, and power spectrum of an average image from 20 aligned images.

Traditional structure determination methods other than electron microscopy, such as NMR and X-ray crystallography, can generally not be applied to the structure of these large macromolecular complexes, with a few spectacular exceptions such as the ribosome (Yusupov et al., 2001) and RNA polymerase (Cramer et al., 2000). Many biologically highly relevant macromolecules in the cell can only be found in small copy numbers, making biochemical purification procedures difficult and usually not efficient enough to produce complexes in the amount and homogeneity needed for structural studies by X-ray crystallography. As a consequence, there is an increasing demand for $3 \mathrm{D}$ structure determination of these complexes by single-particle electron cryomicroscopy. Single-particle cryo-EM structures of large macromolecules can add significantly to our understanding of the architecture, dynamics and function of macromolecular complexes even at intermediate levels of resolution. Of course, there is a justified hope that routine structure determination at higher resolution is possible and has so far only been limited for practical reasons (Henderson, 1995). However, one of the major difficulties in singleparticle cryo-EM is not the refinement of an available structure to obtain higher resolution, but rather the structure determination de novo of a molecule. It is thus important to start de novo structure determination with images that are of the best quality obtainable.

Whether the use of images recorded on a CCD camera helps in improving the image phases at low-tomedium resolutions was therefore tested in this work. As we show, the quality of the image phases of low $(\sim 20 \AA)$ spatial frequencies can be improved by about $8^{\circ}$ as estimated from analysing images of amorphous carbon film. The fact that phases of CCD images are more reliable adds significantly to the understanding of the better performance of CCD-recorded images in single-particle analysis. On the basis of the results of the comparison 

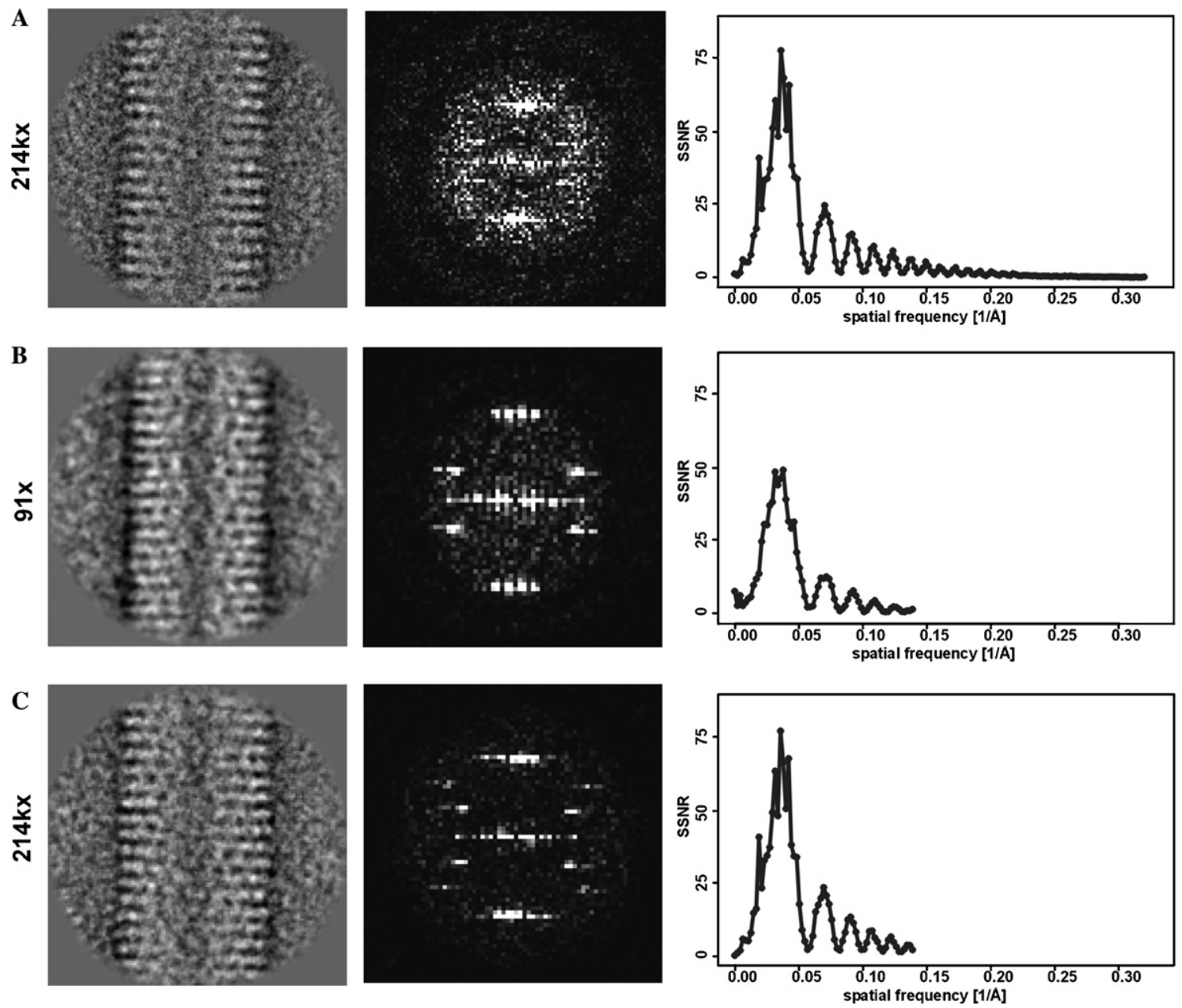

Fig. 9. Influence of the sampling interval on the performance of alignment and classification. (A) Class average of eight images of TMV recorded at $1.5 \AA$ /pixel as in Fig. 7, fourth row. The power spectrum of the class average shows clearly visible background noise at $\sim 20 \AA$, although the SSNR at this resolution should be very high (right column, results obtained from eight images of carbon film that have been treated identically to the TMV images). (B) Class average of eight images of TMV recorded at $3.5 \AA$ /pixel as in Fig. 7, first row. The power spectrum shows a low level of background noise, the first maximum of the SSNR is about 50\% lower, too. (C) Results obtained with the images shown in (A), but binned in Fourier space by a factor of 2.3 so that the Nyquist frequency becomes identical to that in (B) and oversampling is reduced. In contrast to (A), the alignment and averaging algorithms are able to amplify the helical signal in the 23-18 A range. The high SSNR is retained, leading to a better visual image quality compared with (B).

between digital and analogue image recording, the use of a CCD detector instead of conventional photographic film can clearly be recommended for making initial image-processing in single-particle analysis more reliable. The results obtained in our tests show that the CCD camera is superior in all relevant aspects, and they confirm our practical experience that CCD images are better for de novo structure determination. This general statement agrees well with results that we have obtained using other cameras such as a TVIPS $1 \mathrm{k} \times 1 \mathrm{k}$ (BioCam0124) and TVIPS $2 \mathrm{k} \times 2 \mathrm{k}$ (TemCam F224HD) camera for some of the above mentioned experiments using a few comparable camera settings (data not shown). A possible explanation for better image phases of CCD detectors compared to film may be due to the thickness variations in the emulsion over large regions of the film. Such variations are automatically eliminated by the application of flatfielding combined with dark field subtraction in case of the CCD camera. The higher consistency of image phases is associated with higher SSNR of CCD detectors at low resolutions in comparison with film. Recently reported results on the SNR of CCD cameras in comparison with film (Booth et al., 2004) are in good agreement with our results. In addition, the present work also takes into account the influence of image-processing, by applying alignment algorithms to the image data.

The continuous signal obtained from the carbon film alone makes such films ideal specimens for the kind of tests performed here (Zhang et al., 2003). The scattering properties of amorphous carbon are similar to those of biological specimens, the alignment of carbon-film images always works perfectly, and the signal is equally distributed over the total bandwidth of the recording 
device. Therefore, the various results obtained by operating the camera in different modes can be used to determine the optimum settings for image-recording in single-particle cryo-EM.

A more routine method to test the performance of a recording device is the determination of the modulation transfer function (MTF) from averages of Fourier-transformed electron-noise images (de Ruijter and Weiss, 1992). The MTF has the value one at the base frequency of the device, whereas for all other spatial frequencies up to the Nyquist frequency the relative signal strength is plotted. This approach is especially suitable for the evaluation of the higher-resolution information, whereas differences close to the origin of the MTF are not accentuated. A similar situation is encountered in connection with the B factors (Fig. 4): in fact, the data presented here for signal decay of spatial frequencies appear to imply that the film is superior to all settings of the CCD camera. However, the direct measurement of phase consistency at low-to-intermediate resolutions, using real-world specimens, provides an excellent explanation for the superior quality of images recorded on a CCD detector.

When the performances of different media are being discussed, the interference of the recording device and microscope settings should also be addressed: a large area of the recording device such as a film measuring $9 \times 12 \mathrm{~cm}$ also requires a large diameter of the electron beam in the image plane, leading to longer exposure times and possibly a stronger influence of unavoidable specimen movement. If the brightness of the beam is adjusted by opening the condenser apertures, the advantage of a shorter exposure time is unfortunately cancelled out by the lower coherence of the beam and, thus, higher spatial signal decay. With film, this problem can be overcome by the traditional small-spot scanning procedure (Bullough and Henderson, 1987; Downing, 1991). In contrast, the full area of current CCD chips has to be exposed in practice to avoid time-consuming reading of dead areas. Two-fold binning of the CCD pixels (in the present study leading to an effective pixel length of $30 \mu \mathrm{m}$, twice the $15 \mu \mathrm{m}$ for the unbinned settings) leads to a combination of three effects: (1) 4-fold increased light sensitivity, allowing shorter exposure times and maximum beam coherence, (2) approximately 4-fold increased readout speed and (3) lower internal readout noise. These effects are of course even stronger for $4 \times$ binning, when in total 16 pixels are connected to one "super-pixel" of a side length of $60 \mu \mathrm{m}$. In our experience, the combination of these three effects, in particular the extraordinarily high light sensitivity of CCD detectors, is also responsible for the dramatic improvement of image quality when non-FEG microscopes are used.

An important disadvantage of CCD pixel binning is that the imageable area becomes very small $(\sim 2000 \times 2000 \AA)$, especially when high magnifications are applied at the same time. The situation becomes even worse at higher defoci, where image information is delocalised: if a defocus of $20000 \AA$, a high tension of $200 \mathrm{kV}$ and a spherical aberration constant of $2.0 \mathrm{~mm}$ are assumed, then the $10 \AA$ information of a given point is distributed around a circle of $\sim 50 \AA$ radius, while the $5 \AA$ information is spread over an area of $\sim 100 \AA$ radius. So a globular particle $300 \AA$ in diameter would need a circular area of at least $500 \AA$ to be recorded, in order to allow a complete defocus correction. If the particle is too close to the edge of the image, it is therefore to be excluded, and the useful chip length is reduced by about $10 \%$. On the other hand, CTF effects do not argue against the use of particles close to the edges in the initial, low-resolution $2 \mathrm{D}$ and $3 \mathrm{D}$ analyses.

In practice, the simplest method to record large continuous areas is a variant of small-spot scanning over adjacent areas using the electron microscope's image and beam shift (Sherman et al., 1996). The CCD control software EM-MENU (see above) allows images to be recorded with slight overlap in both the $x$ and the $y$ direction and then to be stitched by a translational 2D cross-correlation algorithm. As a main advantage, manual or (semi-) automated particle selection is faster with only a few large images than with many small ones. As a main disadvantage, small overlap zones with doubled electron dosage occur, owing to the overlap and to the fact that a circular beam has to illuminate a square CCD detector up to the edges of the detector. Furthermore, the use of overlapping image parts is not applicable to native cryo-specimens in ice holes, as the signal of the carbon film is required to determine the exact relative image positions.

The application of the different camera settings to TMV reveals the important influence of the sampling interval. The low-frequency spectrum of an image is known to be important to direct the alignment of the higher spatial frequencies (Shaikh et al., 2003). However, as an intrinsic feature of cross-correlation based algorithms, a large amount of high-frequency noise will misdirect cross-correlation-based alignments to a certain extent and lead to poorer alignment of lower spatial frequencies. For best results in the low- to medium

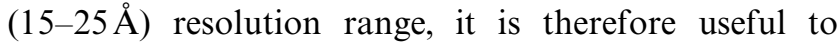
record "oversampled" CCD images and then to coarsen the images to Nyquist frequencies of around $7 \AA$ (see Fig. 9). As expected, and as further demonstrated by the carbon-film experiments, the high SNR of the lower spatial frequencies is unaltered by this procedure. Moreover, interpolation is not necessary, as upon Fourier transformation of the image only the central part of the transform can be reverse-transformed to yield any desired sampling interval. A more sophisticated way to avoid the influence of high-frequency noise is the use of a spatial weighting scheme to assess the similarity of particle and reference images. The weakness of traditional cross-correlation-based alignment procedures to deal 
with high-frequency noise, and the applicability of weighted correlation coefficients, have recently been reviewed in detail (Stewart and Grigorieff, 2004). Overall, the results obtained with improper sampling intervals illustrate that the advantages of higher image quality can easily disappear if over-weighting of the high-frequency components of the images occurs during the initial calculations.

\section{Conclusions}

Our results demonstrate the excellent qualities of images recorded with CCD detectors when the low-tomedium resolution signal $(10-25 \AA)$ is to be recovered from the superposed noise in the initial steps of imageprocessing. This feature is helpful in de novo structure determination of single particles and in other low-contrast situations, e.g., when small protein complexes are imaged and computer processing of the images is difficult. The use of CCD cameras for single-particle structure determination is therefore recommended in the initial steps of the analysis in such situations. In contrast, the current generation of $4 \mathrm{k} \times 4 \mathrm{k}$ CCD cameras does not perform better than conventional silver halide film in situations where molecular resolutions beyond $7 \AA$ are to be reached. However, the performance of the CCD detector depends strongly on the settings of the electron microscope and of the detector itself. Binning of the CCD pixels thus leads to larger effective pixel sizes, with higher SNRs and better transmission of image phases, while the high sensitivity of the CCD camera enables the use of small condenser apertures for maximum coherence of the electron beam. Because for these settings the imageable area becomes very small, the images should be recorded with automated small-spot scanning, as implemented in the CCD camera control software.

\section{Acknowledgments}

We are grateful to Dr. Richard Henderson for his comments on the manuscript. The work was supported by grants from the Bundesministerium für Bildung und Forschung (BMBF) (031U215B, 0331899).

\section{References}

Aloy, P., Böttcher, B., Ceulemans, H., Leutwein, C., Mellwig, C., Fischer, S., Gavin, A.C., Bork, P., Superti-Furga, G., Serrano, L., Russell, R.B., 2004. Structure-based assembly of protein complexes in yeast. Science 303, 2026-2029.

Booth, C.R., Jiang, W., Baker, M.L., Zhou, Z.H., Ludtke, S.J., Chiu, W., 2004. A 9 angstroms single particle reconstruction from CCD captured images on a $200 \mathrm{kV}$ electron cryomicroscope. J. Struct. Biol. $147,116-127$.
Brink, J., Chiu, W., 1994. Applications of a slow-scan CCD camera in protein electron crystallography. J. Struct. Biol. 113, 23-34.

Bullough, P., Henderson, R., 1987. Use of spot-scan procedure for recording low-dose micrographs of beam-sensitive specimens. Ultramicroscopy 21, 223-230.

Bullough, P.A., Henderson, R., 1999. The projection structure of the low temperature $\mathrm{K}$ intermediate of the bacteriorhodopsin photocycle determined by electron diffraction. J. Mol. Biol. 286, 16631671.

Cramer, P., Bushnell, D.A., Fu, J., Gnatt, A.L., Maier-Davis, B., Thompson, N.E., Burgess, R.R., Edwards, A.M., David, P.R., Kornberg, R.D., 2000. Architecture of RNA polymerase II and implications for the transcription mechanism. Science 288, 640-649.

de Ruijter, W.J., Weiss, J.K., 1992. Methods to measure properties of slow-scan CCD cameras for electron detection. Rev. Sci. Instrum. 63, 4314-4321.

Downing, K.H., 1991. Spot-scan imaging in transmission electron microscopy. Science 251, 53-59.

Downing, K.H., Hendrickson, F.M., 1999. Performance of a 2k CCD camera designed for electron crystallography at $400 \mathrm{kV}$. Ultramicroscopy $75,215-233$.

Dube, P., Tavares, P., Lurz, R., van Heel, M., 1993. The portal protein of bacteriophage SPP1: a DNA pump with 13-fold symmetry. EMBO J. 12, 1303-1309.

Fan, G.Y., Ellisman, M.H., 2000. Digital imaging in transmission electron microscopy. J. Microsc. 200 (Pt. 1), 1-13.

Faruqi, A.R., Subramaniam, S., 2000. CCD detectors in high-resolution biological electron microscopy. Q. Rev. Biophys. 33, 127.

Forler, D., Kocher, T., Rode, M., Gentzel, M., Izaurralde, E., Wilm, M., 2003. An efficient protein complex purification method for functional proteomics in higher eukaryotes. Nat. Biotechnol. 21, 89-92.

Frank, J., 1996. Three-Dimensional Electron Microscopy of Macromolecular Assemblies. Academic Press, San Diego.

Gavin, A.C., Bosche, M., Krause, R., Grandi, P., Marzioch, M., Bauer, A., Schultz, J., Rick, J.M., Michon, A.M., Cruciat, C.M., et al., 2002. Functional organization of the yeast proteome by systematic analysis of protein complexes. Nature 415, 141-147.

Golas, M.M., Sander, B., Will, C.L., Lührmann, R., Stark, H., 2003. Molecular architecture of the multiprotein splicing factor SF3b. Science 300, 980-984.

Golas, M.M., Sander, B., Will, C.L., Lührmann, R., Stark, H., 2005. Major conformational change in the complex SF3b upon integration into the spliceosomal U11/U12 di-snRNP as revealed by electron cryomicroscopy. Mol. Cell 17, 869-883.

Henderson, R., 1995. The potential and limitations of neutrons, electrons and X-rays for atomic resolution microscopy of unstained biological molecules. Q. Rev. Biophys. 28, 171-193.

Joyeux, L., Penczek, P.A., 2002. Efficiency of 2D alignment methods. Ultramicroscopy $92,33-46$.

Jurica, M.S., Moore, M.J., 2003. Pre-mRNA splicing: awash in a sea of proteins. Mol. Cell 12, 5-14.

Penczek, P., Radermacher, M., Frank, J., 1992. Three-dimensional reconstruction of single particles embedded in ice. Ultramicroscopy $40,33-53$.

R-Software, 2004. R: A language and environment for statistical computing (R Foundation for Statistical Computing).

Saad, A., Ludtke, S.J., Jakana, J., Rixon, F.J., Tsuruta, H., Chiu, W., 2001. Fourier amplitude decay of electron cryomicroscopic images of single particles and effects on structure determination. J. Struct. Biol. 133, 32-42.

Sander, B., Golas, M.M., Stark, H., 2003a. Automatic CTF correction for single particles based upon multivariate statistical analysis of individual power spectra. J. Struct. Biol. 142, 392-401.

Sander, B., Golas, M.M., Stark, H., 2003b. Corrim-based alignment for improved speed in single-particle image processing. J. Struct. Biol. 143, 219-228. 
Shaikh, T.R., Hegerl, R., Frank, J., 2003. An approach to examining model dependence in EM reconstructions using cross-validation. J. Struct. Biol. 142, 301-310.

Sherman, M.B., Brink, J., Chiu, W., 1996. Performance of a slow-scan $\mathrm{CCD}$ camera for macromolecular imaging in a $400 \mathrm{kV}$ electron cryomicroscope. Micron 27, 129-139.

Sherman, M.B., Chiu, W., 1997. Reliability of phases retrieved from $400-\mathrm{kV}$ spot-scan images of purple membranes acquired on a slowscan CCD camera. J. Microsc. 188 (Pt. 3), 285-289.

Stewart, A., Grigorieff, N., 2004. Noise bias in the refinement of structures derived from single particles. Ultramicroscopy 102, 67-84.

Stewart, P.L., Cary, R.B., Peterson, S.R., Chiu, C.Y., 2000. Digitally collected cryo-electron micrographs for single particle reconstruction. Microsc. Res. Tech. 49, 224-232.

Subramaniam, S., Lindahl, M., Bullough, P., Faruqi, A.R., Tittor, J., Oesterhelt, D., Brown, L., Lanyi, J., Henderson, R., 1999. Protein conformational changes in the bacteriorhodopsin photocycle. J. Mol. Biol. 287, 145-161.
Unser, M., Trus, B.L., Steven, A.C., 1987. A new resolution criterion based on spectral signal-to-noise ratios. Ultramicroscopy 23, 39-52.

van Heel, M., 1984. Multivariate statistical classification of noisy images (randomly oriented biological macromolecules). Ultramicroscopy $13,165-183$.

van Heel, M., Frank, J., 1981. Use of multivariate statistics in analysing the images of biological macromolecules. Ultramicroscopy 6, 187-194.

van Heel, M., Harauz, G., Orlova, E.V., Schmidt, R., Schatz, M., 1996. A new generation of the IMAGIC image processing system. J. Struct. Biol. 116, 17-24.

Yusupov, M.M., Yusupova, G.Z., Baucom, A., Lieberman, K., Earnest, T.N., Cate, J.H., Noller, H.F., 2001. Crystal structure of the ribosome at 5.5 Å resolution. Science 292, 883-896.

Zhang, P., Borgnia, M.J., Mooney, P., Shi, D., Pan, M., O'Herron, P., Mao, A., Brogan, D., Milne, J.L., Subramaniam, S., 2003. Automated image acquisition and processing using a new generation of $4 \mathrm{~K} \times 4 \mathrm{~K} \mathrm{CCD}$ cameras for cryo electron microscopic studies of macromolecular assemblies. J. Struct. Biol. 143, 135-144. 